\title{
The disk property. A short survey
}

\author{
Cezar Joiţa
}

\begin{abstract}
We present some results obtained over the years regarding the disk property for complex manifolds and its connections with pseudoconvexity.
\end{abstract}

The idea to use holomorphic disks to study domains of holomorphy in $\mathbb{C}^{n}$ goes back all the way to F. Hartogs [12] at the beginning of the twentieth century. Hartogs' result was extended by Osgood [19] who proved what is called "Hartogs extension theorem" stating that if $\Omega$ is a domain in $\mathbb{C}^{n}, K \subset \Omega$ is a compact subset and $\Omega \backslash K$ is connected then any holomorphic function on $\Omega \backslash K$ can be extended to $\Omega$. Osgood proof of Hartogs' theorem is incomplete. For a complete proof along Hartogs' ideas see [17]. The shortest and the most elegant proof is due to Ehrenpreis [9] and uses the $\bar{\partial}$-method. Actually Hartogs extension theorem holds in a far more generally context, namely for cohomologically $(n-1)$-complete normal complex spaces, see [4], [18], and [20].

A few years after Hartogs' paper, E. E. Levi [16] founded the theory of pseudoconvexity and proved, using holomorphic disks, that a domain of holomorphy with smooth boundary must be pseudoconvex. Here we say that $\Omega$ is pseudoconvex if it has a strictly plurisubharmonic (i.e. its Levi form, or its complex Hessian, is positive definite) exhaustion function.

Of course, by the Oka's solution to the Levi problem, a domain in $\mathbb{C}^{n}$ is a domain of holomorphy if and only if it is pseudoconvex.

One of the basic notions needed to deal with holomorphic disks for domains in $\mathbb{C}^{n}$ is the continuity principle. We denote by $\Delta$ the open unit disk in $\mathbb{C}$ and, in general, by $\Delta_{r} \subset \mathbb{C}$ the disk of radius $r$ centered at the origin.

Key Words: Stein spaces, holomorphically convex spaces, disk property.

2010 Mathematics Subject Classification: 32E05, 32E10, 32F10.

Received: August, 2011.

Accepted: February, 2012. 
Definition 1. A domain $\Omega$ in $\mathbb{C}^{n}$ satisfies the continuity principle if for every continuous function $F: \bar{\Delta} \times[0,1] \rightarrow \mathbb{C}^{n}$ such that $z \rightarrow F(z, t)$ is holomorphic in $\Delta$ for every $t \in[0,1]$ if $F(\bar{\Delta} \times\{0\}) \subset \Omega$ and $F(\partial \Delta \times[0,1]) \subset \Omega$ then $F(\bar{\Delta} \times[0,1]) \subset \Omega$.

This notion is an "extrinsic " one because $F$ is allowed to take values outside $\Omega$. We want to formulate an equivalent definition that can be extended to complex manifolds (or complex spaces). For $\epsilon>0$ we define $H_{\epsilon} \subset \mathbb{C} \times \mathbb{R}$ as

$$
H_{\epsilon}=\Delta_{1+\epsilon} \times[0,1) \bigcup\{z \in \mathbb{C}: 1-\epsilon<|z|<1+\epsilon\} \times\{1\} .
$$

Definition 2. A complex manifold $X$ is said to satisfy the continuous disk property if whenever $\epsilon$ is a positive number and $F: H_{\epsilon} \rightarrow X$ is a continuous function such that, for every $t \in[0,1), F_{t}: \Delta_{1+\epsilon} \rightarrow X, F_{t}(Z)=F(z, t)$, is holomorphic we have that $F\left(H_{\epsilon_{1}}\right) \Subset X$ for any $\epsilon_{1} \in(0, \epsilon)$.

It is not difficult to see that Definitions 1 and 2 are equivalent for domains in $\mathbb{C}^{n}$.

If the parameter space is the set of positive integers instead of $[0,1]$ we obtain the notion of "discrete disk property".

Definition 3. We say that a complex manifold $X$ satisfies the discrete disk property if for every sequence of functions $f_{n}: \bar{\Delta} \rightarrow X, n \in \mathbb{N}$, continuous on $\bar{\Delta}$ and holomorphic on $\Delta$, if $\bigcup_{n \in \mathbb{N}} f_{n}(\partial \Delta) \Subset X$ then $\bigcup_{n \in \mathbb{N}} f_{n}(\bar{\Delta}) \Subset X$.

A continuous function $f: \bar{\Delta} \rightarrow X$ which is holomorphic on $\Delta$ is called a holomorphic disk in $X$. We remark that in [6] the discrete disk property was called $p_{5}$-convexity. In general controlling the behavior of a sequence of holomorphic disks only on $\partial \Delta$ might not give sufficient information for its behavior in $\Delta$. Take, for example, $f_{n}: \bar{\Delta} \rightarrow \mathbb{C}, f_{n}(z)=z^{n}$, which converges uniformly to 0 on compacts subsets of $\Delta$ but diverges at every point of $\partial \Delta$. We introduce then a weaker notion:

Definition 4. We say that a complex manifold $X$ satisfies the weak discrete disk property if whenever $f_{n}: U \rightarrow X$ is a sequence of holomorphic functions defined on an open neighborhood $U$ of $\bar{\Delta}$ for which there exists an $\epsilon>0$ and a continuous function $\gamma: S^{1}=\{z \in \mathbb{C}:|z|=1\} \rightarrow X$ such that $\Delta_{1+\epsilon} \subset U$, $\bigcup_{n \geq 1} f_{n}\left(\Delta_{1+\epsilon} \backslash \Delta\right)$ is relatively compact in $X$ and $f_{n \mid S^{1}}$ converges uniformly to $\gamma$ we have that $\bigcup_{n \geq 1} f_{n}(\bar{\Delta})$ is relatively compact in $X$.

We should mention that we do not actually know any example showing that the weak discrete disk property is not equivalent to the discrete disk property. Obviously the weak discrete disk property implies the continuous one.

The proof of the next Lemma is, more or less, straightforward. 
Lemma 1. If $X$ is a complex manifold, $\left\{D_{n}\right\}$ is an increasing sequence of open subsets of $X$ such that $\bigcup D_{n}=X$ and if each $D_{n}$ satisfies the continuous disk property then $X$ satisfies the continuous disk property.

J. E. Fornæss [10] gave an example of a complex $X$ manifold that can be written as an increasing union of Stein open subsets and $X$ does not satisfy the weak discrete property. By the previous lemma, $X$ satisfies the continuous disk property. This shows that the weak discrete disk property is stronger that the continuous one.

For domains in $\mathbb{C}^{n}$ all this notions of "disk properties" are equivalent among them and they are equivalent to pseudoconvexity. The same type of result holds for Stein manifolds. This is not true anymore on Stein complex spaces (hence if one allows singularities). For examples in this sense, see [5] and [11].

To prove that the disk property implies pseudoconvexity one shows that, for a domain $\Omega$ that satisfies the disk property, $-\log \delta_{\Omega}$ is plurisubharmonic on $\Omega$ where $\delta_{\Omega}$ is the distance to the boundary of $\Omega$. It turns out that one can use the distance to the boundary to produce plurisubharmonic functions for some other classes of complex manifolds.

Definition 5. A complex manifold is called infinitesimally homogeneous if the global holomorphic vector fields generate the tangent space at every point.

For a domain $\Omega$ in an infinitesimally homogeneous manifold $X$ one can define a distance to the boundary $\delta_{\Omega}$ using trajectories of global holomorphic vector fields. A. Hirschowitz [13] proved the following:

Theorem 1. If $X$ is an infinitesimally homogeneous manifold and $\Omega$ is a domain in $X$ that satisfies the discrete disk property then $-\log \delta_{\Omega}$ is plurisubharmonic.

Of course one can endow the complex manifold $X$ with a hermitian metric $\omega$ and for a domain $\Omega \subset X$ one can define $\delta_{\Omega}^{\omega}$ to be the distance to the boundary of $\Omega$ with respect to this metric. However without some extra geometric assumptions this will not be very useful. For Kähler manifolds with positive bisectional curvature Elencwajg [8] and Suzuki [24] have proved the following theorem (see also [25]):

Theorem 2. Let $X$ be a Kähler manifold and $\omega$ be a Kähler metric on $X$. Assume that the biholomorphic bisectional curvature of $\omega$ is positive. If $\Omega$ is an open subsets of $X$ that satisfies the discrete disk property then there exists a neighborhood $U$ of $\partial \Omega$ such that $\delta_{\Omega}^{\omega}$ is strictly plurisubharmonic on $U \cap \Omega$.

Another method to produce plurisubharmonic functions using analytic disks is by using disk functionals. This theory was initiated by E. A. Poletsky. For a complex manifold $X$ let $\mathcal{A}_{X}$ be the set of all holomorphic disks 
in $X$ and let $H: \mathcal{A}_{X} \rightarrow[-\infty, \infty]$ be a function. The envelope of $H$ is defined as $E H: X \rightarrow[-\infty, \infty], E H(p)=\inf \left\{H(f): f \in \mathcal{A}_{X}, f(0)=p\right\}$. The main problem is to prove that $E H$ is plurisubharmonic for various choices of $H$. We refer to [21], [22], [14], [15], and [23] for various results in this direction.

We will discuss next the discrete disk property for coverings of complex surfaces. One motivation for studying this type of problems is the Shafarevich conjecture which asks to decide if universal covering of a projective manifold is holomorphically convex. A complex manifold $X$ is called holomorphically convex if for every compact subset $K \subset X$ its holomorphically convex hull $\hat{K}:=\left\{x \in X:|f(x)| \leq\|f\|_{K} \forall f \in \mathcal{O}(X)\right\}$ is compact. $\|f\|_{K}$ denotes the supremum of $|f|$ on $K$ and $\mathcal{O}(X)$ the set of holomorphic functions on $X$. By the maximum modulus principle every holomorphically convex manifold satisfies the discrete disk property. In its full generality Shafarevich conjecture is open even for surfaces. For partial results we refer to [7].

Definition 6. A complex manifold $X$ is called 1-convex if there exists a normal Stein space $Y$, a proper holomorphic map $p: X \rightarrow Y$ and a finite subset $A$ of $Y$ such that $p: X \backslash p^{-1}(A) \rightarrow Y \backslash A$ is a biholomorphism.

Suppose that $X$ is a 1-convex surface and $p: \tilde{X} \rightarrow X$ is a covering map. It was proved in [1] that $\tilde{X}$ can be exhausted by a sequence of relatively compact strongly pseudoconvex domains with smooth boundary and therefore it satisfies the continuous disk property. Hence the question is if $\tilde{X}$ satisfies or not the discrete disk property. We want to present two results: one in the positive direction and one in the negative direction. To formulate the first one of them we need one more definition:

Definition 7. Let $L$ be a connected 1-dimensional complex space and $\cup L_{i}$ be its decomposition into irreducible components. $L$ is called an infinite Nori string if all $L_{i}$ are compact and $L$ is not compact

The following theorem was proved in [2].

Theorem 3. Let $X$ be a 1-convex surface and $p: \tilde{X} \rightarrow X$ be a covering map. If $\tilde{X}$ does not contain an infinite Nori string of rational curves then $\tilde{X}$ satisfies the discrete disk property.

To explain the condition that $\tilde{X}$ does not contain an infinite Nori string of rational curves that appears in Theorem 3 we mention the following Lemma:

Lemma 2. If $L$ is 1-dimensional complex space that does not contain an infinite Nori string of rational curves then $L$ has a holomorphically convex covering space. 
It turns out that the condition is really necessary because in general $\tilde{X}$ might not satisfy the weak discrete disk property (and therefore nor the discrete disk property) as the next theorem, proved in [3], shows.

Theorem 4. There exists a 1-convex surface whose universal covering does not satisfy weak the discrete disk property.

To prove this theorem one constructs an example. The construction is as follows: we start with $\mathbb{C}^{2}$ and perform, step by step, an infinite sequence of blow-ups. The center of each blow-up is chosen to be a point on the exceptional divisor of the previous blow-up. We do the same type of construction going back-wards in order to obtain an infinite Nori string $L$, all its irreducible components being biholomorphic to $\mathbb{P}^{1}$, indexed after $\mathbb{Z}$. We blow-up each irreducible component of $L$ at a point and we consider $L_{1}$ to be the proper transform of $L$. If we choose the centers of all these blow-ups appropriately we can show that a small enough neighborhood of $L_{1}$ does not satisfy the weak discrete disk property and covers a 1-convex surface.

Acknowledgments : This work was partially supported by CNCS grant PNII-ID-PCE-2011-3-0269.

\section{References}

[1] M. Colţoiu: Coverings of 1-convex manifolds with 1-dimensional exceptional set. Comment. Math. Helv. 68 (1993), no. 3, 469-479.

[2] M. Colţoiu; C. Joiţa: The disk property of coverings of 1-convex surfaces. Proc. Amer. Math. Soc. 140 (2012), no. 2, 575-580.

[3] M. Colţoiu; C. Joiţa: Convexity properties of coverings of 1-convex surfaces. Preprint, arXiv:1110.5791v1.

[4] M. Colţoiu; J. Ruppenthal: On Hartogs' extension theorem on $(n-1)$ complete complex spaces. J. Reine Angew. Math. 637 (2009), 41-47.

[5] M. Colţoiu; M. Tibăr: On the disk theorem. Math. Ann. 345 (2009), no. $1,175-183$.

[6] F. Docquier; H. Grauert: Levisches Problem und Rungescher Satz für Teilgebiete Steinscher Mannigfaltigkeiten. Math. Ann. 140 (1960), 94123

[7] P. Eyssidieux; L. Katzarkov; T. Pantev; M. Ramachandran: Linear Shafarevich Conjecture. Preprint, arXiv:0904.0693. 
[8] G. Elencwajg: Pseudo-convexité locale dans les variétés kahlériennes. Ann. Inst. Fourier (Grenoble) 25 (1975), no. 2, 295-314.

[9] L. Ehrenpreis: A new proof and an extension of Hartogs' theorem. Bull. Amer. Math. Soc. 67 (1961), 507-509, .

[10] J. E. Fornæss: 2 dimensional counterexamples to generalizations of the Levi problem. Math. Ann. 230 (1977), no. 2, 169-173.

[11] J. E.Fornæss; R. Narasimhan: The Levi problem on complex spaces with singularities. Math. Ann. 248 (1980), no. 1, 47-72.

[12] F. Hartogs: Einige Folgerungen aus der Cauchyschen Integralformel bei Funktionen mehrerer Veränderlichen, Bayerische Akademie der Wissenschaften. Mathematish-Physikalisch Klasse, 36 (1906), 223-292.

[13] A. Hirschowitz: Pseudoconvexité au-dessus d'espaces plus ou moins homogènes. Invent. Math. 26 (1974), 303-322.

[14] F. Lárusson; R. Sigurdsson: Plurisubharmonic functions and analytic discs on manifolds. J. Reine Angew. Math. 501 (1998), 1-39.

[15] F. Lárusson; R. Sigurdsson: Plurisubharmonicity of envelopes of disc functionals on manifolds. J. Reine Angew. Math. 555 (2003), 27-38.

[16] E.E. Levi: Studii sui punti singolari essenziali delle funzioni analitiche di due o più variabili complesse. Annali di Matematica Pura e Applicata, 17 (1910), 61-87.

[17] J. Merker; E. Porten: A Morse-theoretical proof of the Hartogs extension theorem. J. Geom. Anal. 17 (2007), no. 3, 513-546.

[18] J. Merker; E. Porten: The Hartogs extension theorem on $(n-1)$-complete complex spaces. J. Reine Angew. Math. 637 (2009), 23-39.

[19] W.F. Osgood: Lehrbuch der Funktionentheorie, Bd II, B.G. Teubner, Leipzig, (1929).

[20] N. Øvrelid; S. Vassiliadou: Semiglobal results for $\bar{\partial}$ on complex spaces with arbitrary singularities, Part II. Trans. Amer. Math. Soc. 363 (2011), no. $12,6177-6196$

[21] E. A. Poletsky: Plurisubharmonic functions as solutions of variational problems, Several complex variables and complex geometry (Santa Cruz, CA, 1989), Proc. Sympos. Pure Math., 52, Part 1, Amer. Math. Soc., 1991, pp. 163-171. 
[22] E. A. Poletsky: The minimum principle. Indiana Univ. Math. J. 51 (2002), 269-303.

[23] J.-P. Rosay: Poletsky theory of disks on holomorphic manifolds. Indiana Univ. Math. J. 52 (2003), no. 1, 157-169.

[24] O. Suzuki: Pseudoconvex domains on a Kähler manifold with positive holomorphic bisectional curvature. Publ. Res. Inst. Math. Sci. 12 (1976/77), no. 1, 191-214.

[25] A. Takeuchi: Domaines pseudoconvexes sur les variétés kählériennes. $J$. Math. Kyoto Univ. 6 (1967), 323-357.

Cezar JOIŢA,

Institute of Mathematics of the Romanian Academy,

P.O. Box 1-764, Bucharest 014700, Romania.

Email: Cezar.Joita@imar.ro 
